\title{
INDUCTION HEATING TOPOLOGY WITH ASSYMETRICAL SWITCHING SCHEME
}

\author{
Janet Teresa K. Cyriac ${ }^{1}$, Sreekala $\mathbf{P}^{2}$

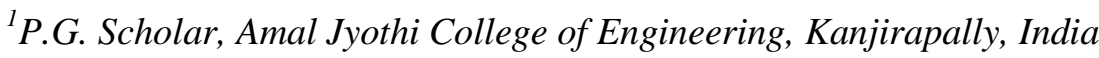 \\ ${ }^{2}$ Assistant Professor, Amal Jyothi College of Engineering, Kanjirapally, India
}

\begin{abstract}
Domestic induction appliances require power converters that feature high efficiency and accurate power control in a wide range of operating conditions. To achieve this, modulation techniques play a key role to optimize the power converter operation. A softswitching high-frequency resonant inverter for induction heating applications is used here. Three switches are used in this topology, one switch in the high voltage side and two switches in the low voltage side. The working of the circuit is illustrated by adopting the operating principle of the high frequency resonant inverter in periodic switching equivalent circuit mode. The topology is advantageous compared to full bridge topology since the number of switches is reduced in this topology. The switching stresses are also reduced due to the soft switching operation of the circuit.
\end{abstract}

Keywords-induction heating, high frequency resonant inverter, soft switching $* * *$

\section{INTRODUCTION}

The working of motors and transformers lead to heat losses. The idea of induction heating emerged, when it was noted that the working of motors and transformers produced heat losses. If the loss heat is utilized, properly, it will lead to the improvement in the efficiency of the total system too. The development of high frequency power supplies also paved a way for induction heating and its applications. At the beginning stages, the trial and error methods of using different applications of induction heating were done without the basic knowledge of the principle of the induction heating. Throughout the years the expansion of the basic ideas has lead to such a huge improvement in this area, which even includes the computer modeling of the elements used in the induction heating. Electric heating is superior to other methods of heating since it has no moving parts, since it is clean and efficient and finally it offers a compact and reliable heat system. Electric heating is done possibly in two methods namely high frequency heating and power frequency heating. At power frequency heating, heat is transferred to the material to be heated by the heat transmission methods like conduction, convection or radiation. In high frequency electric heating, electric energy is converted into heat within the material itself. High frequency heating is therefore more efficient. High frequency heating mainly consists of induction heating and dielectric heating. Induction heating is applied to heat conducting materials like metals. Induction heating is the process of heating the metals by inducing an electric current to flow in the object to be heated. It is similar to the operation of a transformer, i.e. electromagnetic induction principle by Faraday's high frequency source is used to drive a large alternating current through a coil, called the working coil. Primary current flow induces an e.m.f. or current in the secondary. Surface of the pan gets heated.

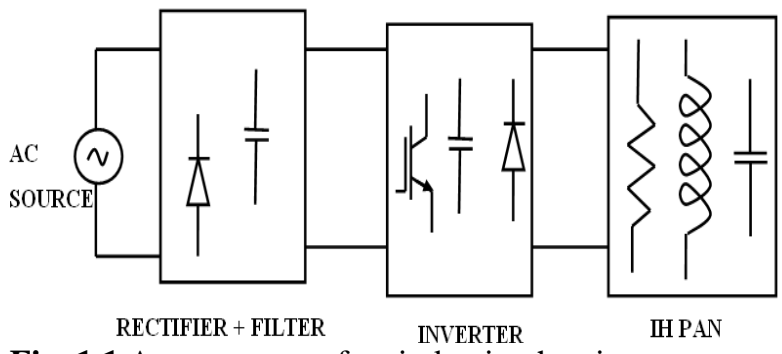

Fig. 1.1 Arrangement of an induction heating system

\section{LITERATURE REVIEW}

\subsection{Series Resonant Inverters}

In a voltage source fed inverter the input voltage is held constant by a capacitor with high capacitance value which maintains the input voltage constant. The series resonant element inductor is connected in series with the capacitor, thus causing series resonant operation in the load circuit. The most commonly used topology for induction heating in series resonant inverters is $\mathrm{H}$ bridge topology. In this configuration two switches in the same leg cannot conduct at the same time. The time delay between the turning on of one of the switches in one arm and another switch in other leg is called dead time. The figure below illustrates a series load resonant circuit and the $\mathrm{H}$ bridge topology used for the circuit. Parallel resonant load circuit, LLC combination type circuits can also be used for induction heating applications. 


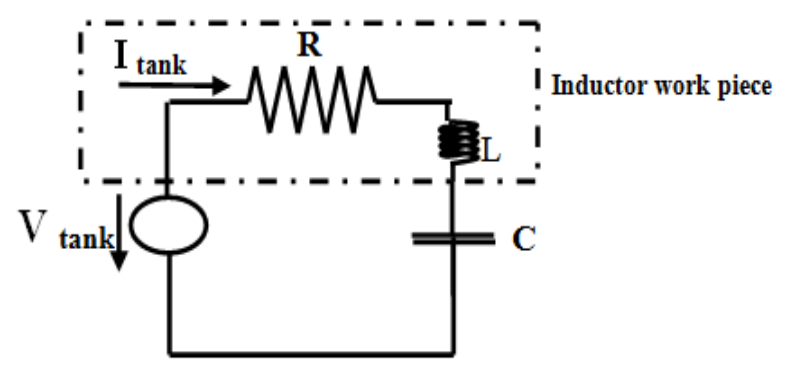

Fig. 2.1 A series tank inductor circuit

\subsection{Full Bridge Configuration of Series Resonant}

\section{Inverter}

In industrial heating of round wires which require less than $5 \mathrm{KW}$ of output power, the configuration used is an $\mathrm{H}$ bridge topology. In most studies the converters are assumed to operate with $50 \%$ duty cycle. The operation of the full bridge resonant topology is similar to that of a full bridge inverter configuration. The circuit diagram of this topology is detailed in the below given figure. The switches $S_{1}$ and $S_{4}$ conduct in the first mode of operation giving rise to an output voltage $\mathrm{V}$ $\mathrm{dc}$ across the load. When the load current tends to reverse, the diodes corresponding to the switches $S_{1}$ and $S_{4}$ conduct for a small delay time, thus making the load current to flow continuously. The same operation continues with the switches $S_{2}$ and $S_{3}$ thus giving an output voltage of $-V_{d c}$ across the output.

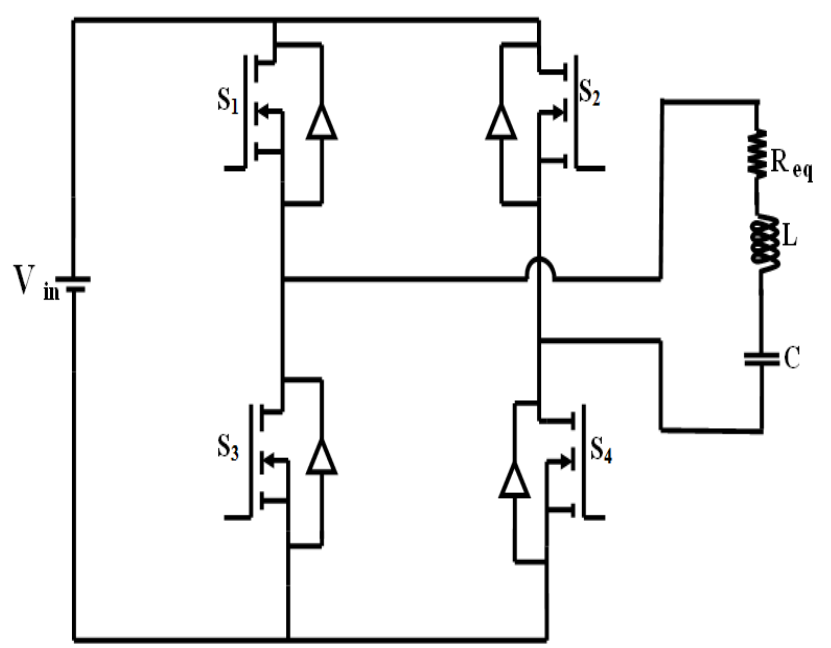

Fig.2.2.The full bridge configuration of series resonant inverter

\subsection{Half Brigde Topology of Series Loaded Resonant}

\section{Inverters}

The half bridge topology also has voltage fed and current fed series and parallel loaded resonant topologies. The main assumptions made in the working of this topology are

1. Resonant capacitors are ideal

2. Inductance $\mathrm{L}$ is of the working coil and the work piece

3. Resistance $\mathrm{R}$ is the resistance of the work piece

4. All devices are lossless
The modes of operation of this configuration are discussed below.

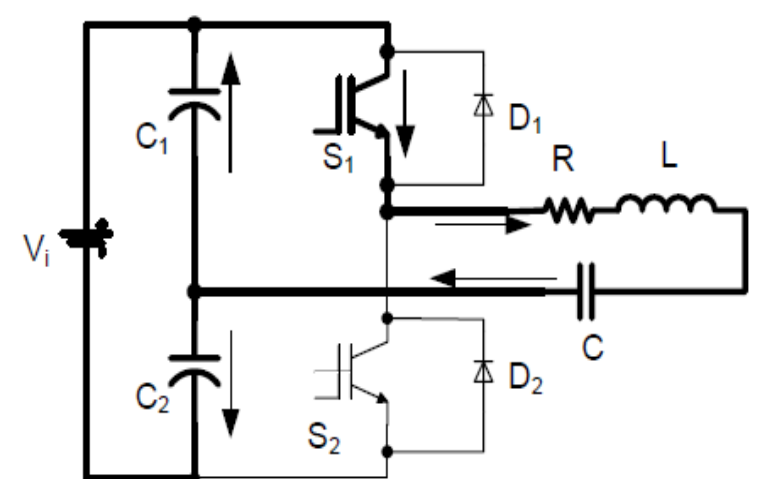

Fig.2.3 The half bridge configuration of series resonant inverter Mode 1

The switch $S_{1}$ conducts load current $i_{L}$. The output voltage across the load is $V_{i} / 2$. When the output current reaches zero and tends to reverse its direction, the diode $\mathrm{D}_{1}$ gets forward biased. Thus the turn off of the switch $S_{1}$ can be achieved at zero current. This can considerably reduce the switching loss during the turn off condition.

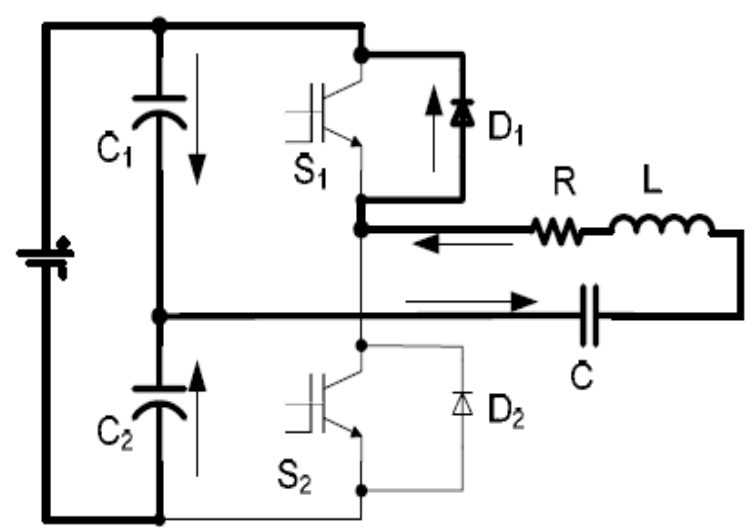

Fig.2.4 The half bridge configuration of series resonant inverter Mode 2

In mode two operation, the forward biased diode $D_{1}$ continues its conduction for a specified time interval.

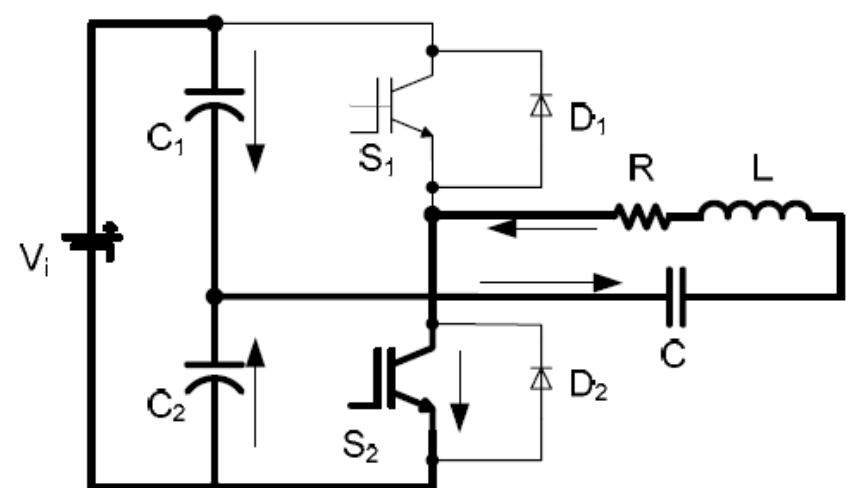

Fig.2.5 The half bridge configuration of series resonant inverter Mode 3 
The switch $\mathrm{S}_{2}$ conducts load current - $\mathrm{i}_{\mathrm{L}}$. The output voltage across the load is $-\mathrm{V}_{\mathrm{i}} / 2$. When the output current reaches zero and tends to reverse its direction, the diode $\mathrm{D}_{2}$ gets forward biased. Thus the turn off of the switch $\mathrm{S}_{2}$ can be achieved at zero current. This can considerably reduce the switching loss during the turn off condition. This operation continues for the entire time range of mode 2 operation.

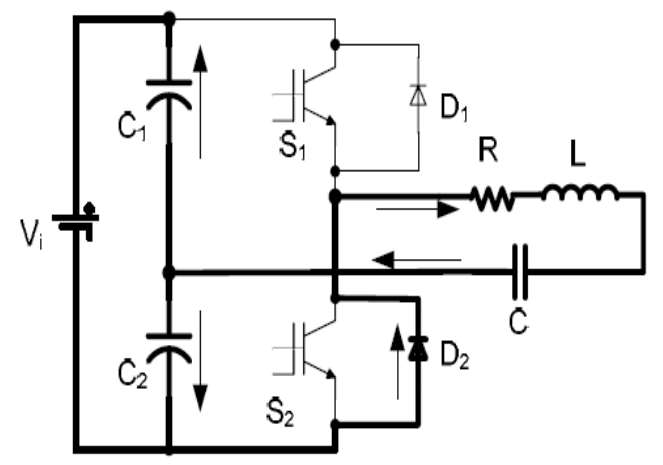

Fig.2.6 The half bridge configuration of series resonant inverter Mode 4

In mode two operation, the forward biased diode $\mathrm{D}_{2}$ continues its conduction for a specified time interval. This section thus gives a brief description about the operation of the full bridge and half bridge inverters used for resonant topologies.

\section{ASYMMETRICAL CONFIGURATION}

The high frequency inverter discussed in this topology consists of a lossless snubber capacitor and an auxiliary switched capacitor. The inverter topology proposed in this topology consists of a dual duty cycle PWM controlled switches under the condition of constant high frequency. The snubber capacitor and the resonant switched capacitor used to describe the modes, has its unique characteristics. The equivalent circuit describes the various modes of operation of this resonant switched topology used commonly in domestic induction heating applications. This topology can also be used in super heated steamer systems, steamers e.t.c.Dual duty cycle PWM is proposed here.

High power conversion efficiency and high frequency A.C. regulation are the improved performance characteristics of this topology. In real the AC grid fed voltage is connected to a rectifier circuit along with a power factor correction circuit before being fed to the resonant inverter. The circuit consists of two switches in one leg and one switch in the other leg of the topology. The high side arm consists of the switch $\mathrm{Q}_{1}$ and the lower side arm consists of the switch $\mathrm{Q}_{2}$ with the parallel lossless snubber capacitor $C_{1}$, the resonant auxiliary switched capacitor $\mathrm{C}_{\mathrm{r} 2}$, the induction heating load represented by the two winding transformer in series with the load resonant capacitor $\mathrm{C}_{\mathrm{r} 1}$. The equivalent circuit of the induction heating load consists of the stainless steel material along with the working coil.
The circuit diagram of the equivalent representation is depicted in the given figures. Latest power electronics has its trend in building equipments for smart home or in building energy management systems. The induction heating appliances uses the topology of either the matrix converters, high frequency resonant converters or high frequency cyclo converters. Among the various topologies of the resonant converters used for induction heating, series loaded, parallel loaded, series parallel loaded or quasi resonant inverters are available.Assymetrical PWM is used for higher power settings range and Resonant switched capacitor PWM is used for lower power settings range. Power regulation, power conversion efficiency characteristics and soft commutation operating range are the considered parameters in this work.

The dual duty cycle PWM mode is operating under the condition of constant high frequency.

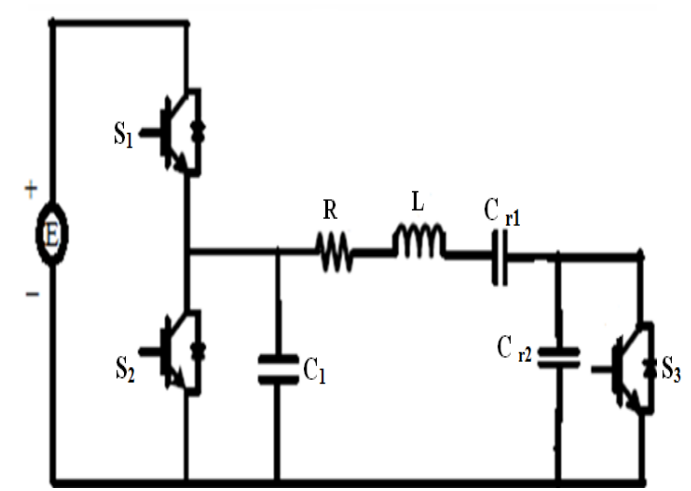

Fig. 3.1 The asymmetrical configuration of IH topology

The switching pulses of the dual duty cycle control scheme are demonstrated below.

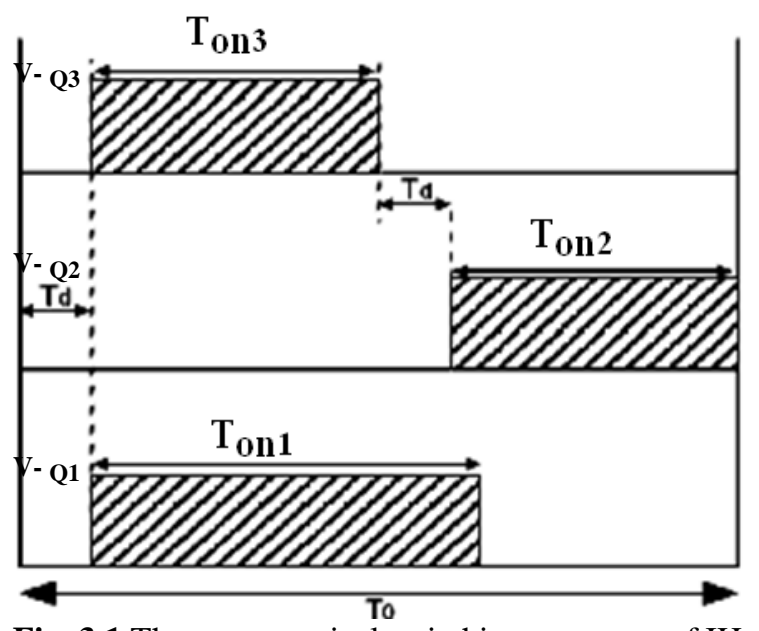

Fig. 3.1 The asymmetrical switching sequence of IH topology

The equivalent modes of operation of the circuit are explained below. There are three modes of operation. Each mode is represented by an equivalent circuit model. 


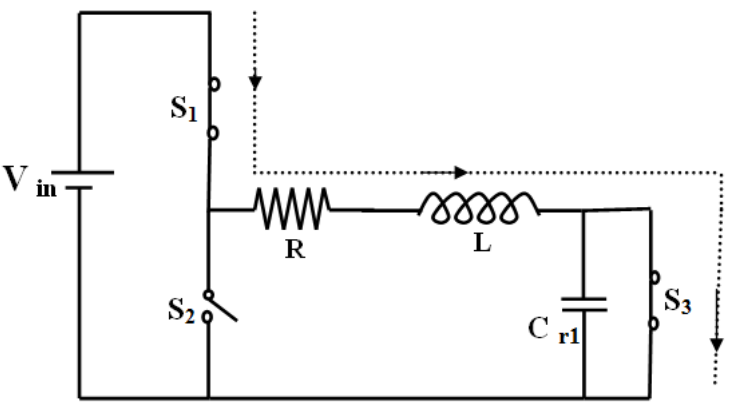

Fig. 3.2 Mode 1 operation

The switch $\mathrm{S}_{3}$ conducts for a time interval $\mathrm{T}_{\text {on } 1}$ followed by a delay time $T_{d}$.During the whole period of $\left(T_{\text {on1 }}+T_{d}\right)$, the switch $S_{1}$ is conducting. Hence in the first mode of operation of the circuit, the current flows from the source, to the switch $\mathrm{S}_{1}$, the IH load and back to the source through the switch $\mathrm{S}_{3}$.If we assume that the resonant capacitor in the IH load discharges through the diodes $\mathrm{D}_{3}$ and $\mathrm{D}_{1}$, it can be assumed that the zero voltage turn off can be achieved for switches $S_{1}$ and $S_{3}$. The snubber capacitor in the low voltage side of the configuration only plays the role for protection of the circuit.

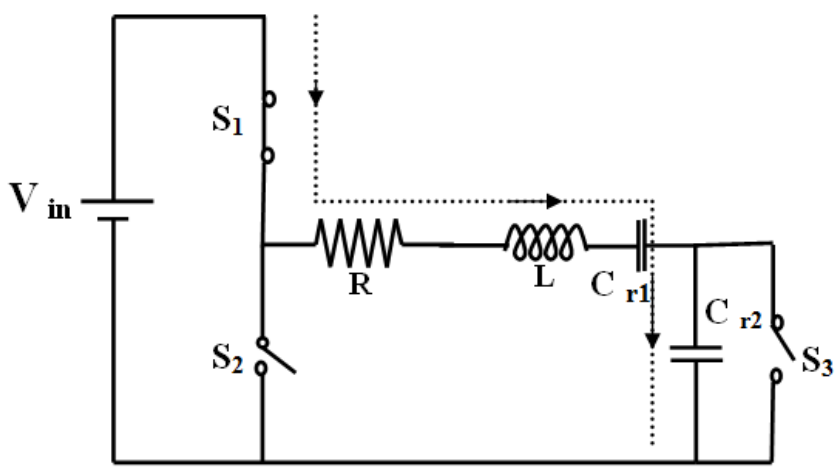

Fig.3.3. Mode 2 operation
In the second mode of operation the switch $S_{1}$ is still conducting for a delay time $t_{d}$. This allows the charging of the series resonant capacitor with positive polarity in the upper plate and negative polarity in the lower plate. This completes the second mode of operation.

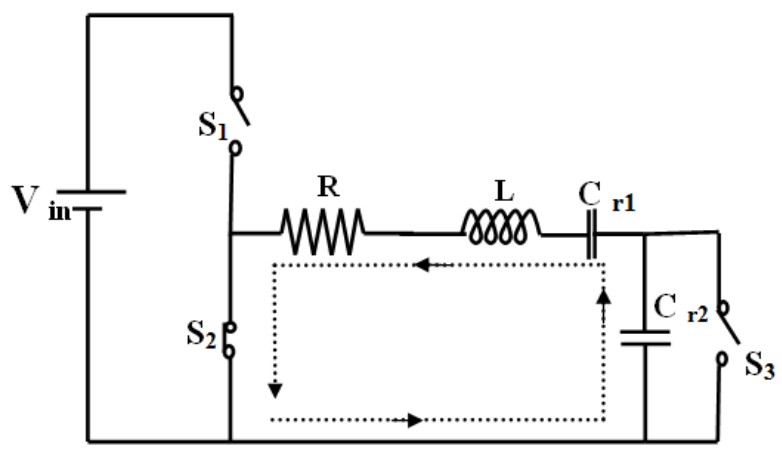

Fig.3.3. Mode 3 operation

In the third mode of operation, the switch $\mathrm{S}_{2}$ is kept on for the time period of $\mathrm{T}_{\mathrm{on} 2}$. The charged capacitor discharges through the switch $\mathrm{S}_{2}$. After the capacitor discharges completely, the reverse biased current flows through diode $\mathrm{D}_{3}$ and switch $\mathrm{S}_{2}$ .Since the turn on of the switch $\mathrm{S}_{3}$ can be done at zero voltage, the switching loss at the turn on condition can be reduced. This completes one cycle of operation. The capacitor $\mathrm{C}_{\mathrm{r} 2}$ acts as a boost capacitor giving an increase in the output voltage and hence output power. This is understood from the simulation results

\section{SIMULATION RESULTS}

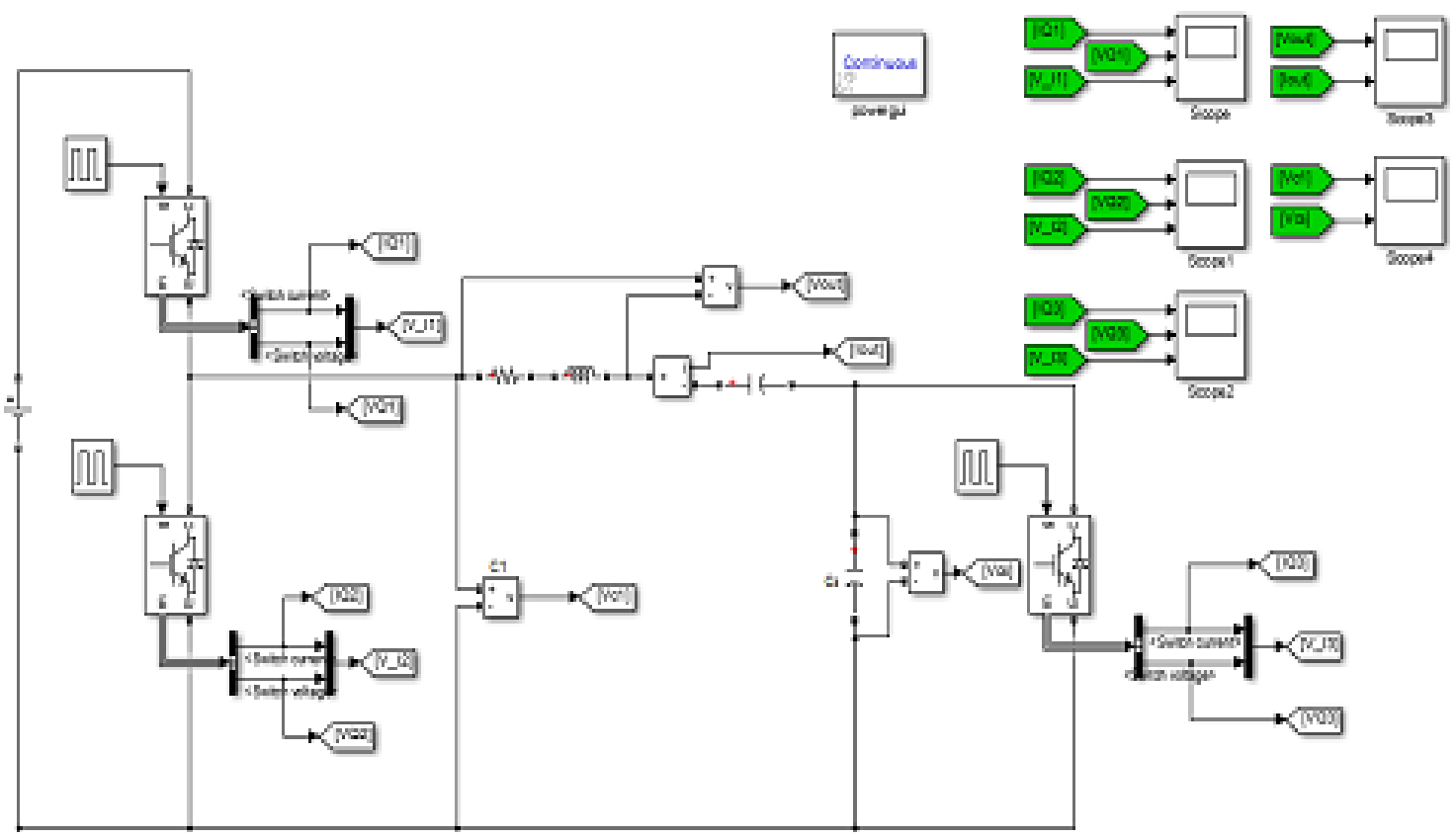

Fig.4.1 Simulink model of the IH system 


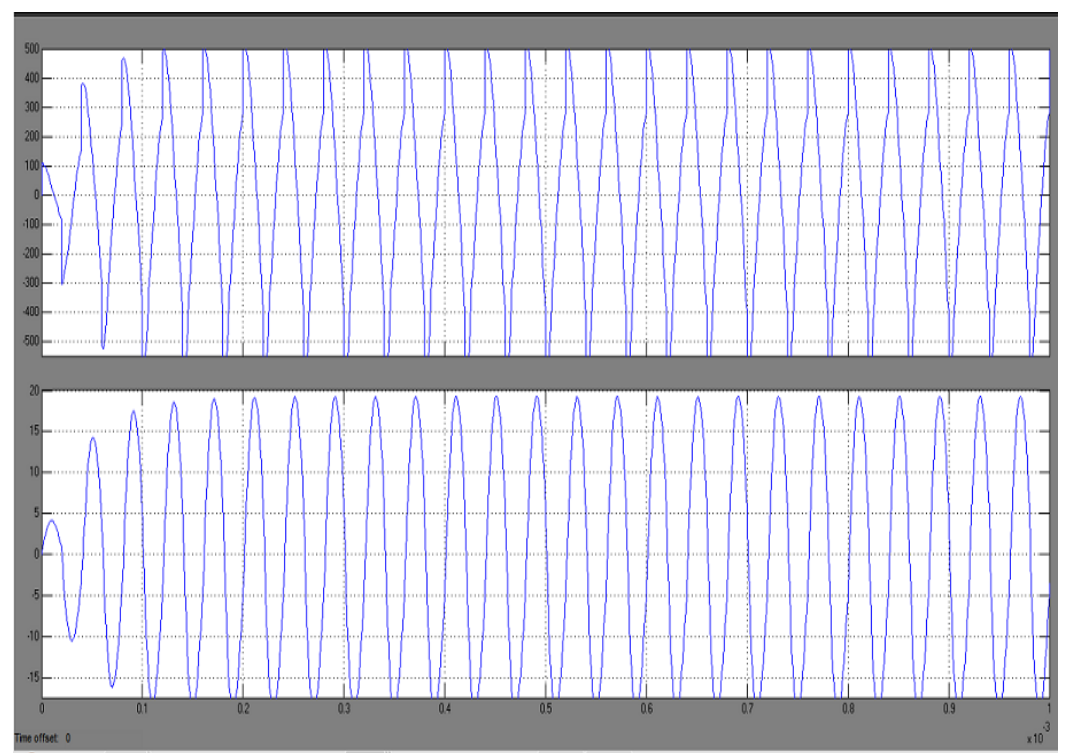

Fig.4.2 Output voltage and current waveforms

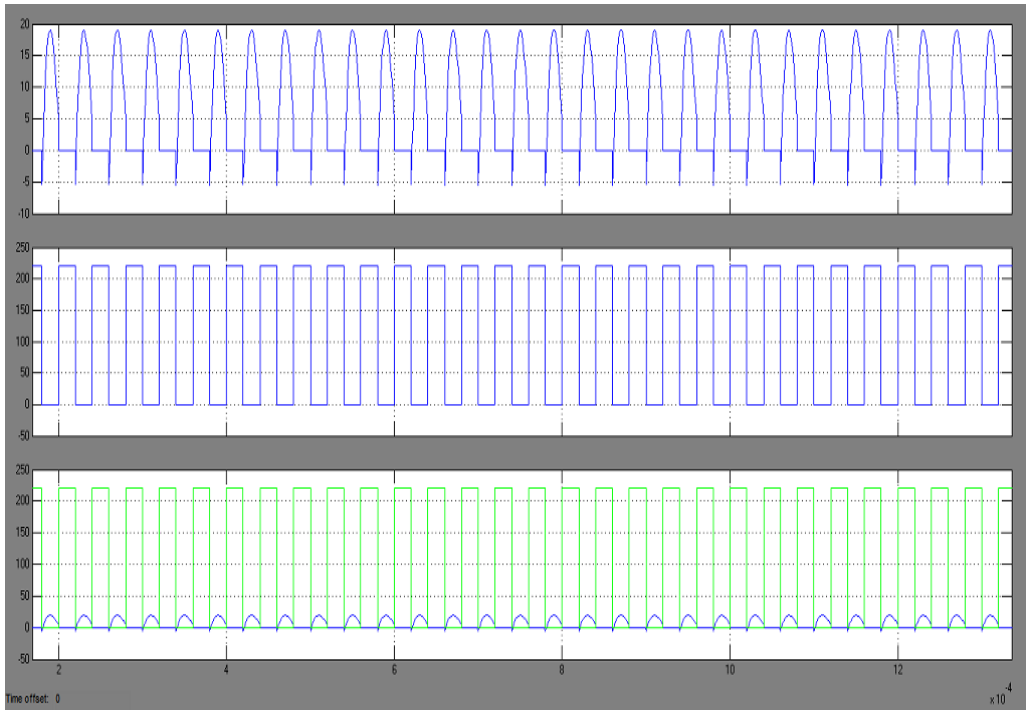

Fig.4.3 Voltage and current waveforms across switch $S_{3}$

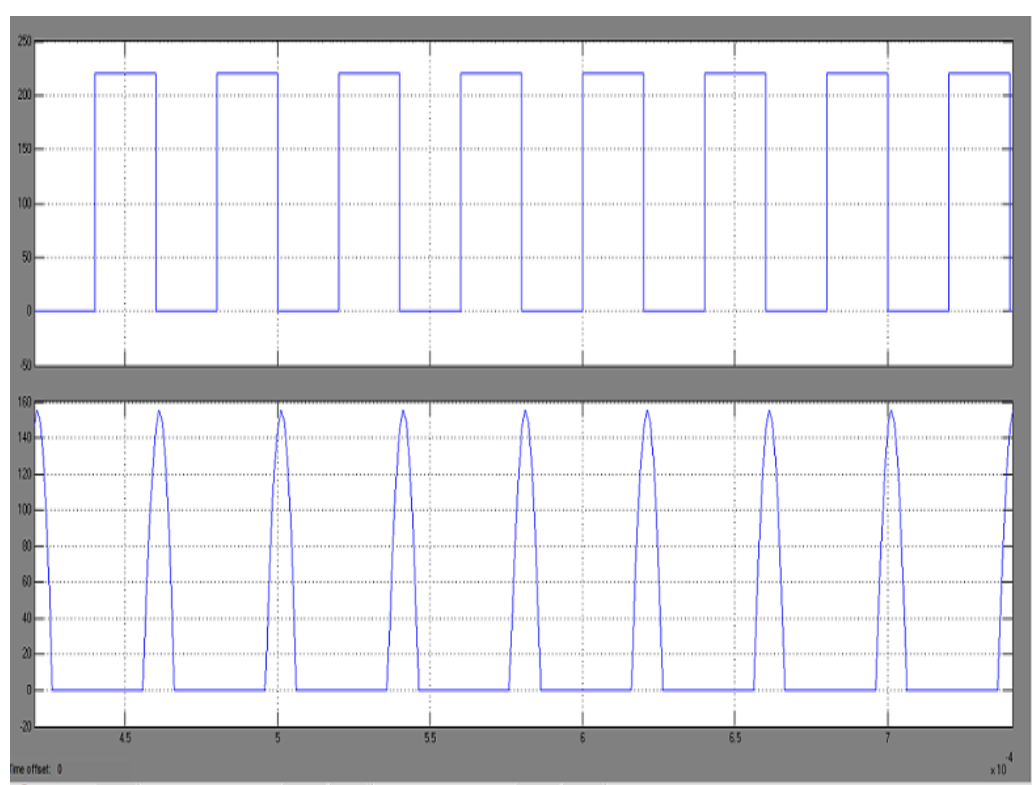

Fig.4.4 Voltage across snubber capacitor and boosting capacitor 


\section{CONCLUSION}

The modes of operation of an induction heating topology with three switches are analyzed brief review on the working of the half bridge and the full bridge topologies are also mentioned. Compared to full bridge and half bridge topology, this configuration provides an output power boosting. Since the number of switches are less, the switching losses can be reduced compared to the full bridge topology. The simulation results of the output voltage can verify the slight boosting capability provided by the energy storing capacitor. Thus a simple and advantageous topology of Induction Heating has been studied in brief.

\section{REFERENCES}

[1] H. P. Ngoc, H. Fujita, K. Ozaki, and N. Uchida, "Phase angle control of high frequency resonant currents in a multiple inverter system for induction heating," IEEE Trans. Power Electron., 26,(11), pp. 3357-3366, Nov. 2011.

[2] H. Kifune, Y. Hatanaka and M. Nakaoka, "Cost effective phase shifted pulse modulation soft switching high frequency inverter for induction heating applications" IEE Proc.-Electr. Power Appl., 151(1), pp. 19-25, January 2004.

[3] Bishwajit Saha and Rae-Young Kim "High Power Density Series Resonant Inverter Using an Auxiliary Switched Capacitor Cell for Induction Heating Applications", IEEE Transactions on Power Electronics, 29 (4), 1-3, April 2014.

[4] P K Banerjee, Bishwajit Saha, Hyun Woo Lee and Mutsuo Nakaoka, "Dual duty cycle controlled softswitching high Frequency inverter using auxiliary reverse Blocking switched resonant capacitor", 4th International Conference on Electrical and Computer Engineering ICECE 2006, 19-21 December 2006, Dhaka, Bangladesh.

[5] Bishwajit Saha, H. Sugimura, T. Mishima, S. Sumiyoshi,H. Omori, S. P. Mun, and Mutsuo Nakaoka, "Dual pwm controlled soft switching high frequency IH load resonant inverter with lossless snubbing capacitor and switched capacitor", IEEE Proceeding-Electric Power Applications.

[6] B. Saha, K.Y. Suh, S. K. Kwon, T. Mishima, M. Nakaoka,"Selective Dual Duty Cycle Controlled High Frequency Inverter Using Resonant Capacitor in Parallel with Auxiliary Reverse Blocking Switch" Journal of Power Electronics (KIPE), 7 (2), pp 119123, April 2007 